\title{
Migración internacional: actividades agrícolas y distribución del ingreso en una comunidad indígena mexicana*
}

\author{
International Migration, Agricultural Activities and income \\ distribution in a Mexican indigenous community
}

Migração internacional, atividades agrícolas e distribuição do
ingresso numa comunidade indígena mexicana

\section{Renato Salas Alfaro** México}

Recibido el 14 de abril de 2011 - aprobado el 2 de mayo de 2011

\section{Resumen}

Objetivos. Este trabajo explora el efecto que tiene la migración internacional y las remesas sobre las actividades agrícolas y la distribución del ingreso en una comunidad indígena de reciente migración internacional. Materiales y método. La información proviene de una encuesta aplicada probabilísticamente en el año 2005, en el 35\% de los hogares. El efecto sobre la distribución del ingreso se estimó mediante la técnica de descomposición del coeficiente de Gini. Los efectos sobre la agricultura se evaluaron con modelos que relacionaron variables agrícolas - demanda de peones, producción, abandono de tierras y ganadería- con factores productivos, socio-demográficos y de financiamiento a la migración en el hogar. Resultados. Los resultados muestran que la migración internacional no afecta la demanda de trabajo agrícola ni la ganadería, pero impulsa la producción agrícola y la recuperación de tierras abandonadas. Conclusión. Las remesas no afectan la demanda de trabajo ni la producción agrícola, pero impulsan la ganadería y la recuperación de tierras, además empeoran de manera marginal la distribución del ingreso total en la comunidad.

Palabras claves: migración internacional, comunidad indígena, actividades agrícolas, distribución del ingreso.

* Trabajo resultado de la tesis doctoral, sustentada en 2007; Instituto Tecnológico de Oaxaca, México.

* * Economista • Ph. D. en Ciencias en Planificación de Empresas y Desarrollo Regional • Centro de Investigación y Estudios Avanzados de la Población, de la Universidad Autónoma del Estado de México•mt13@hotmail.com 


\begin{abstract}
Objectives. This paper explores the effect of international migration and remittances on agricultural activities and income distribution in an indigenous community of recent international migration. Materials and method. The information derives from a probabilistic survey applied in 2005 in 35\% of homes. The effect on income distribution was estimated by means of the method of decomposition of Gini coefficient. The effects on agriculture were evaluated with models that related agricultural variables - demand of workers, production, land abandonment and cattle breeding - and production, sociodemographic and financial factors with migration at home. Results. Results show that international migration does not affect the demand of agricultural work nor cattle breeding, but drive agricultural production and recovery of land abandonment. Conclusion. Remittances do not affect the demand of work nor agricultural production, but drive cattle breeding and land recovery. Additionally, they make worse, in a marginal way, the total income distribution within the community.
\end{abstract}

Keywords: International migration, indigenous community, agricultural activities, income distribution.

\title{
Resumo
}

Objetivos. Este trabalho explora o efeito que tem a migração internacional e as remessas sobre as atividades agrícolas e a distribuição do ingresso numa comunidade indígena de recente migração internacional. Materiais e método. A informação origina se duma pesquisa aplicada probabilisticamente no ano 2005 , a porcentagem $35 \%$ dos lares. O efeito sobre a distribuição do ingresso se estimou mediante a técnica de decomposição do coeficiente de Gini. Os efeitos sobre a agricultura se avaliaram com modelos que relacionaram variáveis agrícolas - demanda de peões, produção, abandono de terras e gado- com fatores produtivos, sócio-demograficos e de financiamento à migração no lar. Resultados. Os resultados mostram que a migração internacional não afeita à demanda de trabalho agrícola nem o gado, mas impulsiona a produção agrícola e a recuperação de terras abandonadas. Conclusão. As remessas não afeitam a demanda de trabalho nem a produção agrícola, mas impulsionam o gado e a recuperação de terras, além empioram de maneira marginal a distribuição do ingresso total na comunidade.

Palavras chave: migração internacional, comunidade indígena, atividades agrícolas e

30 distribuição do ingresso. 


\section{Introducción}

En México, algunas comunidades del centro y del norte del país tienen ya más de un siglo migrando hacia Estados Unidos (EU). Actualmente, es una actividad generalizada que incluye a poco más del $80 \%$ de las localidades mexicanas (Conapo, 2002). No obstante, el beneficio que otorga en ambos países, con mano de obra o con remesas, no la exime de los crecientes traumas que deben enfrentarse en ambos países y que, de forma alarmante, incluyen la muerte y el secuestro de los migrantes*. La migración, en cuanto movimiento de personas, implica a su vez el trasiego de sueños, formas de vida, costumbres, intenciones y, en general, una serie de aspectos que definen y representan al ser humano. En este sentido, la migración internacional tiene consecuencias para los lugares de origen y destino. Éstas son tan diversas que parece imposible dimensionarlas todas, pero esto mismo permite acercarse, por lo menos de manera parcial, a algunos efectos específicos. Dado que los estudios de caso, aunque han dado cuenta de algunos efectos que ejercen la migración y sus remesas a nivel de comunidad, sus resultados son contrastantes y se focalizan en ciertos aspectos. Por otro lado, dada la diversidad de este fenómeno, es imposible que las evidencias encontradas en una comunidad, den cabal cuenta del efecto que tiene la migración en otras comunidades.

Desde los trabajos pioneros realizados en los años setenta, una vertiente de investigación, sobre todo cualitativa, ha sostenido que la migración fomentaba la diferenciación de clases sociales, en función de la desigual recepción de ingresos, ligada al status migratorio de los hogares, mientras que algunos de éstos crecían económicamente, otros se pauperizaban. En estos estudios, resalta la formación de un círculo vicioso de mayor migración en la comunidad de origen. La migración fomenta la concentración de la tierra, el ganado y el capital. Las remesas, por su parte, se destinan al consumo conspicuo. La conclusión final es que la migración internacional es un drenaje de recursos para las comunidades. Algunas investigaciones realizadas a partir de los años 80's destacan el potencial de aprovechamiento productivo de las remesas internacionales en la generación de mejores condiciones de vida en el ámbito local (Adelman, Taylor and Vogel, 1988).

Allí, la migración internacional se percibe como un generador de ingresos, que los hogares pueden utilizar en actividades de producción local. Incluso, señalan que el simple hecho de consumir las remesas forma efectos multiplicadores de ingreso que fomentan, mediante el nuevo consumo, la formación de opciones productivas dentro

* Por ejemplo, las mujeres de Centroamérica en su paso por México, hasta se inyectan anticonceptivos para no quedar embarazadas por violación (Díaz y Kunher, 2007); en el caso de la extorsión y secuestro de estos migrantes, se estima que genera ganancias anuales superiores a los 50 millones de dólares para las bandas del crimen organizado y autoridades de seguridad pública mexicanas; cuando los familiares en EU no pagan, los ponen a vender droga, violan a las mujeres o matan a los migrantes (Ramírez, 2010; Pérez y Cilia, 2010). 
o fuera de la comunidad, según la fortaleza de sus vínculos sectoriales. Con énfasis en los determinantes de la migración, sostienen que las remesas compensan en exceso el drenaje económico inicial de la emigración internacional.

Por su parte, los estudios que han analizado la relación que guardan la migración internacional y las remesas con las actividades agrícolas, en algunos que siguen una visión económica de la migración, se cuantifica en términos económicos y estadísticos dicha relación (Lucas, 1987). Sin embargo, utilizan exclusivamente el "volumen de producción" como variable representativa de toda la actividad agrícola, y dejan de lado otras actividades que se imbrican con éstas (traspatio, recolección de leña, consumo, cuida de animales), y que resulta difícil separar unas de otras, sobre todo en una comunidad indígena. Por otro lado, los estudios que analizan los efectos de las remesas internacionales sobre la distribución del ingreso en México, tienen limitaciones en el tamaño de la muestra. Uno considera una muestra de 30 hogares (Stark, Taylor and Yitzhaki, 1986); otro, aunque contempla una muestra nacional de aproximadamente 1.700 hogares, desagregan el análisis por regiones geográficas del país que, según ellos, representan las etapas migratorias de las comunidades que allí se encuentran (Taylor, Mora, Adams and López, 2005).

\section{Materiales y método}

Este trabajo analizó, con datos obtenidos directamente en los hogares, la forma y la intensidad con la que afectan la migración internacional y las remesas en la distribución del ingreso y sobre las actividades agrícolas en la comunidad de San Miguel del Valle en Oaxaca. Para tal efecto, se levantó una muestra probabilística en 155 hogares que representan el 35\% del total, se aplicó un cuestionario a los jefes de hogar, adicionalmente se realizaron entrevistas a profundidad a diversos actores. El trabajo de campo se realizó en el verano de 2005, por lo cual las cantidades refieren el año 2004, en razón de que la comunidad solamente realiza un ciclo agrícola. El cuestionario obtenía información económica cuantificable y algunos datos etnográficos de los hogares. Éste comprendió cuatro secciones; la sociodemografía del hogar, las actividades agrícolas, las fuentes y montos de ingreso y sus actividades de migración internacional; interrogaba a los jefes de hogar sobre las actividades agrícolas y sus fases, los tipos de trabajo que empleaban y su origen, las diferentes fuentes de ingreso -monetario y en especie- a las que recurren, la historia de la migración del hogar. Asimismo inquiría sobre la autovaloración hacia sus activos - casas, ganado, autos-, y las formas de financiamiento de la migración internacional en el hogar. La selección de la muestra siguió el procedimiento del muestreo aleatorio simple sin reposición para poblaciones humanas; los resultados que aporta, son estadísticamente validos para realizar inferencias hacia la población total. Una vez determinado el número de hogares, se procedió a su determinación concreta, el marco muestral se conformó por el padrón total de hogares que poseía la agencia municipal y la clínica de salud local. Para la aplicación del cuestionario, fue necesario 
recurrir al apoyo de sus autoridades, quienes inicialmente, condicionaron que no se hicieran promociones religiosas ni políticas a los hogares. Una vez convenidos en el reclamo, el agente municipal y su cabildo autorizaron la realización de entrevistas en los hogares; posteriormente avisaron a éstos con el fin de que cooperaran, que una persona pasaría a aplicarles unas preguntas sobre sus actividades diarias. Se eligió inicialmente un hogar al azar, mediante la elección de un número aleatorio obtenido de la calculadora, con el apoyo de una persona nativa de San Miguel, el hogar fue ubicado en el mapa de la comunidad, el segundo hogar fue elegido, omitiendo los dos hogares que le sucedían, en el orden del padrón de registros.

Los efectos sobre las actividades agrícolas se analizaron mediante un modelo lineal múltiple que relacionó variables agrícolas omitidas en otros trabajos (producción, consumo, ganadería, tierras en abandono y demanda de peones), con factores de producción en el hogar -tierra, capital y trabajo-, y se adicionó un vector del financiamiento de la migración y otro de las características sociodemográficas en el hogar. El efecto sobre la distribución del ingreso se examina con la descomposición del coeficiente de Gini. Ésta desagrega el coeficiente de Gini del ingreso total en tres componentes empíricos: (Sk) que representa la proporción del ingreso de cada fuente en el ingreso total; (Gk) que representa el coeficiente de Gini para cada fuente de ingreso; y (Rk) que representa la correlación Gini de la fuente de ingreso (k) al rango del ingreso total*. Igualmente, considerando que la migración internacional en San Miguel es de reciente masificación, se puede esperar, según los planteamientos teóricos, que funja como un buen contraste para evaluar los efectos en el área agrícola como en la distribución del ingreso.

\section{La comunidad de San Miguel del Valle}

San Miguel es una comunidad zapoteca, localizada a $37 \mathrm{~km}$ de la ciudad de Oaxaca Capital y $9 \mathrm{~km}$ al norte de Tlacolula. Su población total se compone de 2.509 habitantes que viven en la comunidad y de otros 845 que radican en EU. Es una comunidad fundada alrededor del año 1400, aunque su poblamiento inició en los años 70's. Anteriormente,

* Esta técnica sigue un procedimiento basado en la siguiente propiedad: el coeficiente de Gini es expresado como dos veces la covarianza entre el ingreso del pueblo y la función acumulada de sí mismo, dividida por la media del ingreso general, puede ser igual a la suma de las covarianzas de cada fuente del ingreso total y la función acumulada del ingreso total, igualmente dividido entre la media general del ingreso.

$$
G \mathrm{O}=\frac{2 \operatorname{Cov}\left[Y_{0}, F\left(y_{0}\right)^{-}\right.}{\mathrm{m}}=2 \sum_{k=1}^{k} \frac{\operatorname{Cov}\left[Y_{k}, F\left(y_{0}\right)\right]}{\mathrm{m}_{n}}=\sum_{=1}^{\rightarrow} R_{k} G_{k} S_{k} \quad::=\frac{\operatorname{Cov}\left[Y_{k}, F\left(y_{o}\right)\right]}{\operatorname{Cov}\left[Y_{k}, F\left(y_{k}\right)\right\rceil}
$$

Esto permite determinar la proporción del coeficiente del Gini total, que corresponde a cada fuente de ingreso (Lerman and Yitzhaki, 1985). 
los hogares se encontraban dispersos entre los cerros. Cuenta con un jardín de niños, dos escuelas primarias y una tele-secundaria. Cuenta con una clínica de salud, un edificio de la agencia municipal, uno de la oficina de bienes comunales, un templo del siglo XVII, un panteón, un mercado de abastos, un módulo de turismo, una terminal de transporte y una caseta telefónica privada. Casi toda la población habla lengua zapoteca y alrededor del 95\% práctica la religión católica. El 38\% de la PEA comunal se ocupan en el sector primario, $55 \%$ en el sector secundario y $7 \%$ en el terciario; más del $95 \%$ de la PEA ocupada percibe a lo mucho dos salarios mínimos mensuales de ingreso, situación que permite considerar que son auto empleadas o consideradas como ayudantes del padre o esposo, que es quien recibe el ingreso por sus labores del tejido de la lana.

El sistema de gobierno - político y religioso- en la comunidad ese establece por usos y costumbres. Las responsabilidades en la organización administrativa y espiritual se asignan entre los hombres, jefes de familia; los cargos duran un año, inician a la edad de 15 años como policía auxiliar y terminan cuando son designados encargados del templo; una persona cede a la comunidad alrededor de 15 años de su vida cumpliendo cargos; nunca percibe pago; en algunos cargos (salud, forestal, educación), se forman grupos para rolarse días de cumplimiento, de este modo en los días libres laboran sus tierras y telares para obtener su sustento.

\section{Las actividades económicas en la comunidad}

La comunidad practica una serie de actividades económicas, cada una de las cuales tiene su importancia diferenciada, pues el rendimiento económico no decide la preferencia. Por ejemplo, se prefiere la agricultura a la artesanía, aunque ésta aporta mayores ingresos al hogar. La actividad agrícola es muy importante porque en ella participa toda la familia, el producto es propio y va directamente a la alimentación; el tejido, en cambio, normalmente es de alguien más. La artesanía textil es ampliamente practicada en San Miguel, igual que en otras comunidades (Hernández, 2001); aquí se realiza desde 1970, aunque antes se tejía ropa y cobijas sólo para uso personal. Esta práctica ha pasado de padres a hijos, lo que ha generado una habilidad nata, pero este don es aprovechado por los vendedores de Teotitlán del Valle -comunidad vecina-donde se subcontrata a los tejedores de San Miguel para que elaboren prendas que después ellos venden. Éstos financian la artesanía mediante la dotación del hilo, especificación del producto y con préstamos por adelantado. La actividad textil se trabaja en un $66 \%$ de los hogares muestreados; les proporciona poco más del $80 \%$ de sus ingresos salariales, pero sobre todo les representa una actividad para los ratos de ocio, el tiempo estival o el receso migratorio. Igualmente, sirve para mantener ocupados a los hijos, la esposa y los abuelos. Esta es la razón principal de que, siendo una actividad que aporta ingresos al hogar, no sea valorada como actividad primaria, porque los productores no poseen su producto. 
No obstante, a pesar de que el maíz es base de la alimentación zapoteca (De la Fuente, 1998), su importancia está menguando, pues actualmente sólo 70\% de los hogares realiza esta actividad. La retirada de una actividad tan importante se ha visto afectada por la obtención de mayor ingreso mediante la migración internacional, por la mayor disposición de productos básicos a menores precios en tiendas locales, aunque igualmente el tipo de suelo agrícola y la irregularidad de las lluvias han contribuido a desincentivar estas labores. Por ejemplo, una evaluación a precios de mercado del volumen físico de producción del año 2004, versus los costos en que se incurre (considerando y sin considerar el valor de la fuerza de trabajo familiar empleada) muestra que todos los hogares tienen pérdidas económicas en esta actividad. No obstante, entre las razones que empujan a seguir laborando el campo es que estos hogares perciben que la calidad de su grano supera al comprado, además que tener el trabajo acumulado en el hogar no garantiza poder emplearlo por un salario. Por lo tanto, perciben que la actividad agrícola, aunque proporciona poco producto, es un respaldo real.

\section{La migración internacional en San Miguel}

La migración internacional en San Miguel empezó en el programa bracero con la salida de cinco migrantes para EU. Es después de 2000 cuando han partido de la comunidad alrededor del $65 \%$ de los emigrantes totales muestreados. La falta de dominio del idioma español, la obtención de los alimentos de sus cosechas, la ubicación de los hogares en el cerro, las condiciones de pobreza aguda que azotaba a la entidad fueron la razón primaria de que la migración no se masificara durante o inmediatamente después a este programa. Esta situación ocurrió en otras comunidades del centro del país, donde los retornados contaban sus historias y enganchaban a otros. Como se muestra

\section{Gráfica I. Evolución migratoria de San Miguel}

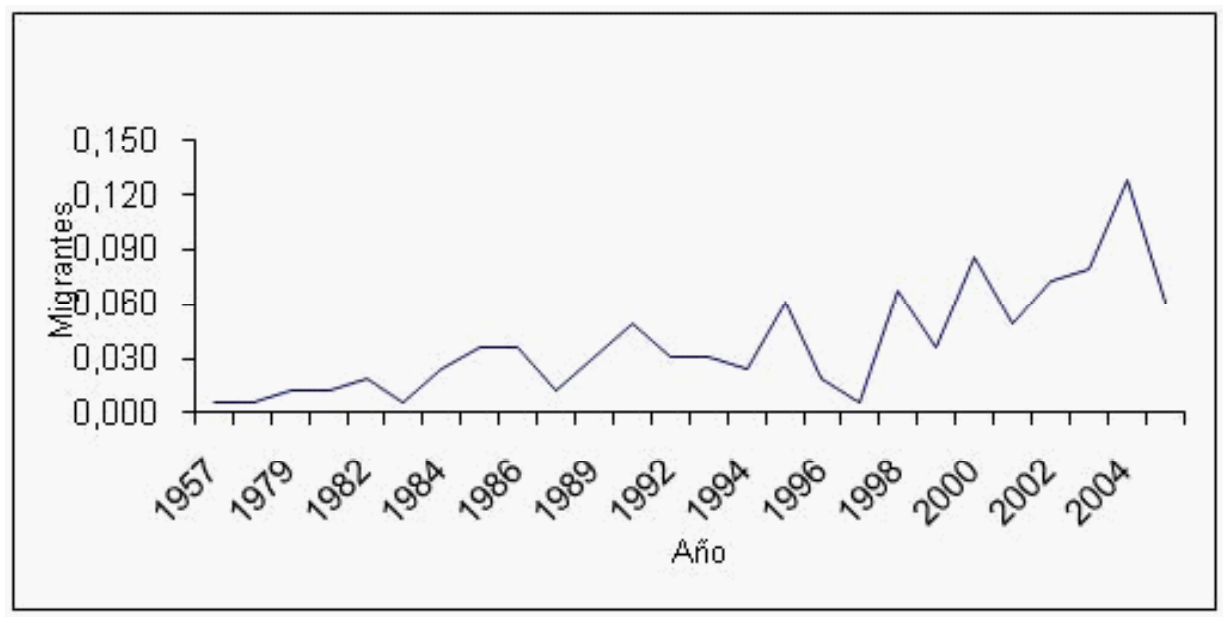

Fuente: elaboración propia. 
(gráfica 1), la masificación migratoria en San Miguel se registra en los años ochenta, concuerda con los tiempos de aplicación en el país de políticas económicas neoliberales y el retiro de apoyos a los pequeños productores, y con el crecimiento de la economía californiana de los años ochenta y noventa, en cuyo nicho de empleos urbanos arribaron los migrantes de San Miguel.

Históricamente, los migrantes de San Miguel han elegido como destino principal Los Ángeles, a donde arriba el 70\% de éstos; Santa Mónica con el 10\%; Las Vegas con 7\%; Colorado, Santa Ana y Pomona con menores porcentajes. A diferencia de las comunidades pioneras del centro y norte del país, donde sus migrantes iniciales eran los jefes de hogar (Mines, 1981; Dinerman, 1982; Reichert, 1981), en San Miguel, son los hijos varones quienes emprenden el recorrido y la masificación. En la migración de San Miguel, alrededor del $75 \%$ de los migrantes son hijos varones, casi todos (94\%) tienen edad entre 14 y 30 años. Desde el principio, se dirigen hacia centros urbanos en busca de empleos relacionados con la cocina, los servicios de aseo en moteles y otros. Esta modalidad se enmarca dentro de lo que se ha llamado la "nueva ola de migración hacia centros urbanos", cuyo origen se ubica primordialmente entre los zapotecos de los Valles Centrales en Oaxaca (Cohen, 2001).

Los migrantes expresan que en la comunidad hay trabajo aunque sea poco y pesado, pero que "bien sirve para irla pasando". Es decir, no migran por falta de recursos económicos para sobrevivir. Más bien, es visualizada por los migrantes como una forma para acumular activos o financiar algunos gastos fuertes. Por ejemplo, las artesanías aportan 1.5 pesos por cada uno proveniente de remesas internacionales; el traspatio aporta 0.65 centavos por cada peso de remesas. El concepto de necesidad económica a que se refieren los migrantes es contextual, según el funcionamiento de los hogares que producen y consumen, se vinculan por lazos de parentesco y compadrazgo. Este enredo social les permite enfrentar con mayor éxito los problemas de subsistencia, por lo que la migración queda para requerimientos mayores, como cuando los jóvenes desean casarse, construir o asumir un cargo. No obstante que ésta les provee recursos y hasta les permite acumular activos o financiar algunas labores de producción, algunas esposas de migrantes expresan que preferirían tener en casa a sus familiares, aunque vivieran peor. En general, de la población que directamente se relaciona con la migración, apenas el 10\% acepta que sus familiares migren, otro $19 \%$ ya se acostumbró, pero al resto no le gusta. Incluso, algunos migrantes ya no desean seguir migrando, pero carecen de apoyos para iniciar su vida localmente. Esto contrasta con lo que afirman algunos sobre la existencia de una gran aceptación y una cultura de migración en Valles Centrales de Oaxaca, y que la migración se realiza como parte de la experiencia cotidiana (Cohen, 2001).

En este caso, es importante la opinión familiar, en razón de que diversos rasgos de la cultura zapoteca hacen que la influencia que ésta tiene sobre las decisiones del migrante sea tan importante, que nadie sale del hogar sin la bendición y las recomen- 
daciones de toda la familia. Por ejemplo, en esta muestra, más del 75\% de los migrantes asume promesas y acuerdos con sus familiares encaminadas al envío de dinero y sobre todo portarse bien, alejarse de los vicios, velar por su seguridad y mantenerse fiel a sus costumbres. Además, transformar un acto individual de decisión en un evento familiar tiene una ventaja económica: el consentimiento de la familia es garantía para gestionar apoyo financiero para los gastos de migración, que aún son caros y que suelen obtenerse del consumo de sus propios ahorros, la venta de activos y, sobre todo, de los préstamos ex profeso. Dado que las deudas en una comunidad se asumen como compromiso familiar, la posibilidad de quedar en peor situación es alta.

Otros rasgos que derivan de la migración internacional y la capacidad económica que ésta provee se aprecian directamente sobre el sistema de cargos. Por ejemplo, existe cierta motivación entre la gente instruida o de posición económica holgada a buscar su inserción en los cargos un poco más arriba de la ordenación tradicional (topil, policía). Asimismo, la comunidad permite que la persona originalmente asignada se libere pagando a otra para que lo cumpla en su persona. En promedio, se pagan $\$ 100$ pesos diarios, o entre $\$ 15.000$ y 18.000 pesos por año, aunque el monto depende del cargo. Esto empezó desde que los migrantes, que no pueden estar presentes en la comunidad, son quienes más pierden al estar un año sin percibir ingreso y porque pueden pagar. Las renuencias de los migrantes influyen para que en las asambleas se acuerde integrar algunas niñas para cumplir el papel de promotoras de salud y otras comisiones menores. Asimismo, en el caso de las mujeres adultas, antes no cumplían cargos pero ahora la comunidad empieza a integrar a las esposas de los migrantes en varias tareas. El argumento es que los esposos ganan dinero y no aportan trabajo.

En la tabla 1 puede apreciarse que, casi el 60\% de los hogares tienen al menos un migrante internacional, alrededor de un cuarto de la población comunal vive en EU, cada familia con migrantes recibe en promedio poco más de 3.500 dólares anuales, que a su vez representan más del 50\% de sus ingresos totales.

\section{Tabla 1. Características seleccionadas de emigración}

\% de familias con migrantes
Promedio de emigrantes por familia
Promedio de edad de emigrantes en su primer viaje
Población de la comunidad que vive en Estados Unidos
Parentesco de migrantes
Promedio de experiencia migratoria del migrante
Dólares gastados en autos
Inversión total de los hogares en migración internacional (años 2000-2004)
Remesas recibidas en promedio en familias con migrantes
\% de las remesas en el ingreso del hogar de los hogares con migrantes
Edad promedio de hogares

$\%$ de familias con migrantes

Promedio de edad de emigrantes en su primer viaje

Población de la comunidad que vive en Estados Unidos

Parentesco de migrantes

Promedio de experiencia migratoria del migrante

Inversión total de los hogares en migración internacional (años 2000-2004)

Remesas recibidas en promedio en familias con migrantes

Edad promedio de hogares

\section{$58 \%$}

1.89

14-30 años (82\%)

$25 \%$

$75 \%$ hijos; $25 \%$ Jefes

6 años

87100 dólares

274000 pesos

3554 dólares

$53 \%$

29.34 años 


\section{$\overbrace{\substack{0 \\ 0}}^{\infty}$ Un poco de teoría}

Como es previsible, se requiere un marco analítico adecuado a las condiciones de San Miguel, que permita, por un lado, la exploración de los efectos que tiene la migración y las remesas sobre la actividad agrícola y sobre la distribución del ingreso. Pero no existe uno que cubra a cabalidad los diferentes aspectos y consecuencias de la migración sobre una comunidad.

No obstante, dado que esta investigación tiene un énfasis socioeconómico, se considera que los planteamientos de la Nueva Teoría Económica de la Migración (NTEM) son una buena aproximación para analizar los efectos de interés, dado que ésta se especializa en el análisis económico de la migración y las remesas sobre las actividades productivas en las localidades rurales*. Aunque igualmente tiene carencias para explicar cualquier impacto empírico que se desee estudiar. Por ejemplo, cuando relaciona remesas con actividades agrícolas o productivas sólo considera el volumen físico de producción. Aún así, la consideración de que las decisiones de migración se toman en el interior del hogar, como una forma de procurarse ingresos que pueden usarse para superar algunas restricciones (crédito, liquidez) que restringen la realización de sus actividades productivas, es aproximada a la situación que atraviesa la toma de decisiones migratorias en el hogar.

En este sentido, la obtención y el uso de las remesas internacionales constituyen la piedra angular que vincula, las causas de la migración con sus impactos. Todo se encuentra mediado por un acuerdo moral mutuo entre migrantes y hogares, lo que asegura la remisión de ingresos al hogar (Lucas and Stark, 1985). Con esta perspectiva, podemos realizar el análisis respecto al hogar y la comunidad en el grupo representativo de hogares.

\section{Resultados}

\section{Migración internacional, remesas y distribución del ingreso en la NTEM}

Los efectos que tienen la migración internacional y las remesas sobre la distribución del ingreso en las comunidades dependen de la historia migratoria. Un trabajo pionero

* El planteamiento general sostiene que cuando una familia rural intenta transformarse hacia la producción comercial, en el proceso enfrentará restricciones: capital para invertir y cobertura de riesgo. Cuando estos mercados fallan, la familia reorganiza sus recursos productivos para buscar fondos y superar esas restricciones, entre sus opciones, la migración se torna muy atractiva dado que puede proporcionar un amplio flujo de ingresos; les incrementa las fuentes y cantidades de ingresos, de modo que el hogar puede realizar sus actividades de producción en las localidades de origen (Stark, 1982). 
argumentaba que las remesas empeoran la distribución del ingreso en las localidades rurales y producen pérdida de bienestar, en razón de que éstas no alcanzan a compensar el efecto adverso de la salida de trabajo y capital para migración, pues la cantidad en que llegan es pequeña y arriba a los hogares de mayor ingreso (Lipton, 1980). Otro trabajo, establece una visión más optimista: sostiene que en los inicios de la migración, pocos hogares se inmiscuyen en ella porque es una etapa costosa, por lo cual los migrantes pioneros suelen provenir del grupo de hogares con mayores ingresos, puesto que se da un efecto negativo sobre la distribución del ingreso. No obstante, una vez que la migración se propaga y los hogares más pobres se inmiscuyen en la corriente migratoria, sus ingresos mejoran y la distribución del ingreso se modifica hacia los más pobres (Stark, Taylor and Yitzhaki, 1986). En un análisis sobre las remesas y la desigualdad en Egipto, se detectó que las remesas del extranjero tienen un efecto negativo en la distribución del ingreso porque son recibidas principalmente por hogares de altos ingresos, que son a su vez quienes envían más migrantes (Adams, 1989). Otros trabajos teóricos proponen la hipótesis de que, si las remesas llegadas a la localidad origen logran rebasar una cantidad crítica, los residentes se beneficiaran de la migración, y lo mismo sucede si ellos no las reciben directamente o si no tienen relación directa con los migrantes remitentes. Analizando una frontera de posibilidades de producción hipotética, señala que la demanda de bienes no comerciables del mercado local de los hogares receptores, crea opciones de comercio a los hogares no receptores, con ello, la reducción de la actividad local por la migración se revierte en el largo plazo y se redistribuyen los ingresos (Djajic, 1986).

\section{Migración internacional, remesas y actividades agrícolas en la NTEM}

La relación que guardan la migración internacional y las remesas con las actividades agrícolas es el tema que más analiza está teoría. No obstante, es el "volumen físico de producción" lo que ha recibido la atención directa (Rozelle, Taylor, and Brauw, 1999). Con ello, omiten actividades agrícolas importantes para el funcionamiento de una comunidad, tales como la demanda de trabajo, el abandono de tierras cultivables, el consumo de granos básicos y la ganadería y los rituales simbólicos y religiosos en que descansan estas actividades. No obstante, aunque esta teoría no analiza estos aspectos, se pueden derivar de ella. La llegada de recursos económicos potencialmente puede generar una mayor demanda de trabajo agrícola y un menor abandono de tierras, aunque mayores recursos también pueden producir menor demanda de granos básicos para alimentación del hogar y menor producción agrícola.

El trabajo pionero plantea que las remesas impulsan los niveles de producción local: directa - gasto concreto- e indirectamente - efectos multiplicadores-. Aunque los efectos directos son pequeños debido a que las comunidades tienen lazos débiles en su vinculación productiva, porque los mercados regionales capturan el gasto de las remesas. 
El efecto indirecto a su vez, resulta del propio gasto de las remesas y de la generación de diversos efectos multiplicadores de consumo que motivan la dinámica de todos los sectores económicos - empleo, ingreso, inversión- en la localidad y la región (Adelman, Taylor y Vogel, 1988). Los autores establecen, mediante una matriz de contabilidad social, que los efectos indirectos en una comunidad rural son positivos pero menores a la unidad. Cada peso adicional de remesas que ingresa motiva los niveles de actividad intersectorial de tal forma que el ingreso por actividades agrícolas aumenta en $\$ 0.417$ unidades adicionales. Por su parte, en el estudio sobre migración e ingresos en localidades de China, se muestra que si bien la salida de trabajadores para migración genera en el corto plazo un efecto negativo sobre el volumen de producción agrícola del hogar, la productividad total del cultivo en cambio se compensa por las remesas en un plazo más largo. Éstas contribuyen a los ingresos del hogar, de tal forma que, por cada unidad adicional que arriba al hogar, aumenta en 0.44 unidades la producción, aunado a que cada migrante nuevo aumenta el ingreso del hogar en 819 unidades monetarias. La conclusión es que las remesas fomentan la productividad agrícola (Rozelle, Taylor and Brauw, 1999). Similar argumento se esgrime para un análisis sobre la emigración de Botswana, Lesotho, Malawi, Mozambique y Sudáfrica, hacia las minas de Sudáfrica y el efecto de este proceso en la producción agrícola de los primeros. Se concluye que en el corto plazo la emigración reduce la producción de cultivos en los sectores de subsistencia de Botswana, Lesotho, Malawi y Sudáfrica, y que, excepto en Lesotho, los ingresos de los migrantes han incrementado la productividad de los cultivos y la acumulación de ganado vacuno en el largo plazo (Lucas, 1987).

Sobre la ganadería, las evidencias muestran que ésta concentra las remesas internacionales como flujo de inversión (Yunez, Taylor y Becerril, 2000). Es decir, los hogares acumulan de este modo ya que les funciona como fondo contingente para tiempos de crisis, y porque el poseer ganado, es un buen principio para generar procesos de acumulación mayores puesto que los migrantes se motivan a enviar más remesas.

La demanda de peones, el abandono de tierras y el consumo doméstico no son aspectos explícitamente estudiados en este enfoque. Y no se tienen evidencias contundentes sobre esta relación. En algunos trabajos previos, encuentran una relación positiva entre remesas y demanda de trabajo agrícola (Acevedo, 2003; Reyes et al., 2001; Cruz, 2002; Tablada y Marrioni, 2003; Rionda, 1992) y en otros se muestra que la migración es más rentable que el trabajo agrícola, porque no se demandan trabajadores agrícolas, pues la salida de migrantes es suplida con los niños, las mujeres y los ancianos del hogar (Dinerman, 1982, p. 72; Wiest, 1983, p. 72). Por su parte, aunque el consumo doméstico no se considera como variable dependiente, en los estudios se mencionan efectos aunque de manera indirecta. En los trabajos de matrices, se muestra que el consumo de productos básicos crece con la llegada de las remesas, pero otro trabajo sostiene que pasa lo contrario, dado que muy poco de las remesas se gasta en la localidad 40 y que la mayoría de productos y alimentos es comprada fuera de ellas (Adelman, Taylor 
and Vogel, 1988; Wiest, 1983, p. 70). En suma, en esta investigación consideramos que el consumo de básicos es una variable primordial (cultural, económica y monetaria) dentro de las actividades agrícolas, puesto que buena parte de éstas, además de las actividades productivas, salariales, donaciones, transferencias y traspatio en general que realizan los hogares, se orienta a la satisfacción de esta necesidad. Para reflejarlo, se analiza en función del planteamiento keynesiano y de la teoría del ciclo vital (Ando, and Modigliani, 1963), considerando como determinantes en el hogar, las remesas, los ingresos corrientes y la riqueza del hogar, más un vector de características socioculturales. Por su parte, el abandono de tierras se agregó en esta investigación para verificar si las remesas contribuyen en el uso productivo de las tierras cultivables. Algunos trabajos pioneros sostienen ampliamente que ésta fomenta la compra de tierras como signo de status, por lo que es retirada de las actividades agrícolas (Dinerman, 1982; Mines, 1981; Reichert, 1981; Wiest, 1979).

\section{Las remesas internacionales y la distribución del ingreso en San Miguel}

La cantidad de ingresos que producen los hogares y su importancia relativa -tabla $2-$, muestra que los salarios y las remesas internacionales acumulan la mayor proporción. En el año de referencia, se generaron \$7.373.277 de ingreso total muestral. Las actividades salariales aportaron $\$ 3.483 .010$ (47\%), de los cuales casi todos fueron generados localmente (apenas 5\% proviene del exterior) y alrededor del 80\% los proveen las actividades artesanales, mientras que por concepto de remesas arribaron alrededor de 216.000 dólares, que, a una tasa de cambio de $\$ 11$, equivalen a $\$ 2.378 .211(32 \%)$. El resto del ingreso se compone de actividades del traspatio, donaciones, regalos, ganadería y el autoconsumo. Como puede notarse, estas actividades otrora estructurales y definitorias de la cultura local, cada vez tienen menor peso.

Una primera aproximación a la forma en que se distribuyen los ingresos, se muestra en las dos columnas de la derecha. Casi tres cuartas partes de los ingresos totales son obtenidas por los hogares que tienen migración internacional, el resto se reparte entre los que no tienen migrantes. Es apreciable que en esta comunidad las remesas son recibidas principalmente por aquellos hogares que ya cuentan de manera importante con otras fuentes de ingreso local. Está situación efectivamente sugiere que las remesas alientan la existencia de una distribución de ingresos sesgada hacia los hogares más ricos, en lugares de origen donde la migración recién comienza a masificarse. Para visualizar esta situación, elaboramos la distribución del ingreso comunal -gráfica 2-. La línea gruesa continua exhibe la manera como se distribuyen los ingresos totales, la línea punteada muestra la distribución de los ingresos domésticos - sin remesas- y la línea delgada de la derecha representa la forma en que se distribuyen las remesas internacionales; la diagonal representa una distribución ideal. Se aprecia una ligera desigualdad 
Tabla 2.Las fuentes de ingreso en San Miguel

\begin{tabular}{|l|l|l|l|l|}
\hline & \multicolumn{2}{|c|}{ La comunidad } & \multicolumn{1}{c|}{$\begin{array}{c}\text { Familias sin } \\
\text { migrantes }\end{array}$} & \multicolumn{1}{|c|}{$\begin{array}{c}\text { Familias con } \\
\text { migrantes }\end{array}$} \\
\hline Fuentes de ingreso (pesos corrientes) & Monto & $\%$ & $\%$ de la fuente & $\%$ de la fuente \\
\hline Ingresos anuales por salarios & 3483010 & $47.24 \%$ & $43.92 \%$ & $56.08 \%$ \\
\hline Dólares anuales de remesas & 216201 & $32.30 \%$ & $0.0 \%$ & $100 \%$ \\
\hline Ingreso anual por transferencias & 265280 & $3.60 \%$ & $34.1 \%$ & $65.9 \%$ \\
\hline Ingreso por negocios & 447400 & $6.07 \%$ & $30.0 \%$ & $70.0 \%$ \\
\hline Ingresos por pagos en especie & 25700 & $0.35 \%$ & $41.24 \%$ & $58.76 \%$ \\
\hline Ingresos por regalos & 23100 & $0.31 \%$ & $5.6 \%$ & $94.37 \%$ \\
\hline Ingresos por retiro de inversiones & 274600 & $3.72 \%$ & $5.4 \%$ & $94.5 \%$ \\
\hline Ingresos por prestamos & 129500 & $1.76 \%$ & $11.0 \%$ & $89.0 \%$ \\
\hline Ahorros anuales del hogar & 93020 & $1.26 \%$ & - & - \\
\hline Ingreso por autoconsumo de leña & 194883 & $2.64 \%$ & $38.98 \%$ & $61.0 \%$ \\
\hline Ingresos de traspatio(frutas) & 26306 & $0.36 \%$ & $22.18 \%$ & $77.8 \%$ \\
\hline Ingresos de traspatio (animales) & 95660 & $1.30 \%$ & $39.55 \%$ & $60.44 \%$ \\
\hline Ingreso agrícola & 29627 & $0.40 \%$ & $0.0 \%$ & $100 \%$ \\
\hline Ingreso total del pueblo & 7373277 & $100.00 \%$ & $26.0 \%$ & $74.0 \%$ \\
\hline
\end{tabular}

Fuente: Elaboración propia

en la distribución de los ingresos comunales, pero las remesas internacionales son las más desigualmente distribuidas entre los hogares de San Miguel; situación comprensible dado que no todos tienen migrantes. Asimismo, es visible que entre los ingresos totales y los domésticos (línea punteada vs línea gruesa), no existe una dominancia,

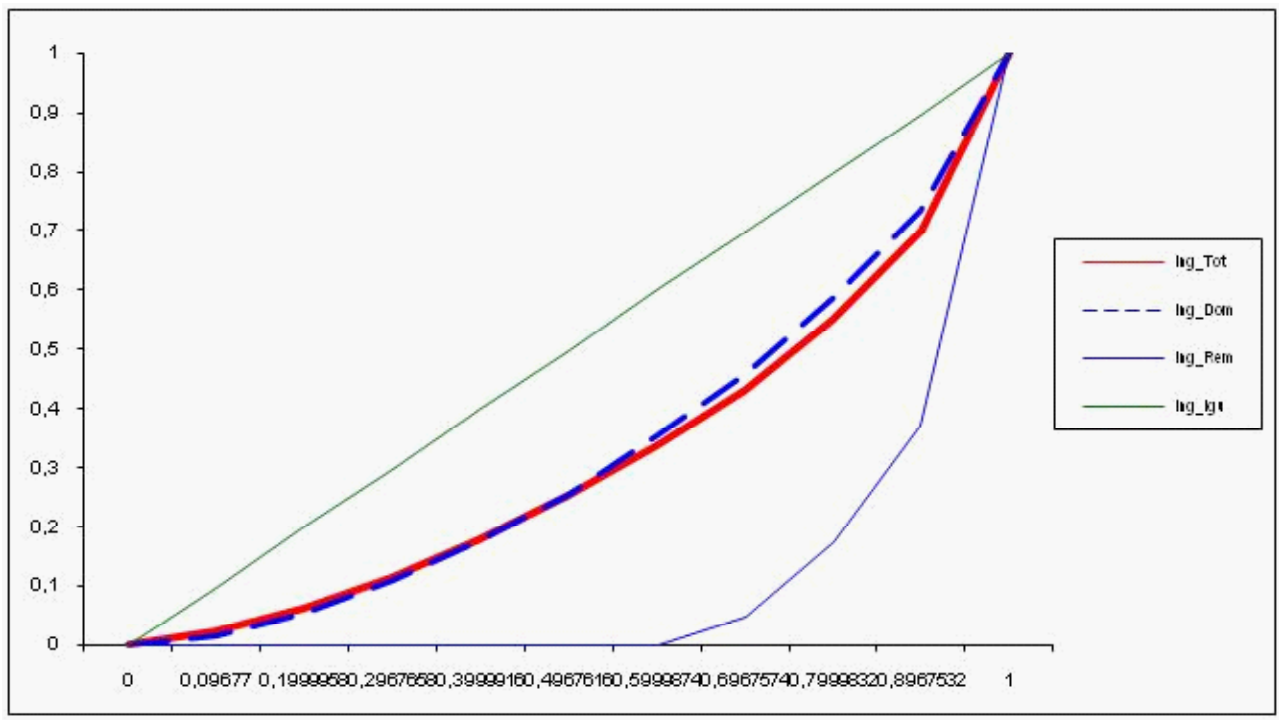

Migración internacional: actividades agricolas y distribución del ingreso en una comunidad indígena mexicana $\bullet$ Renato Salas Alfaro • págs. 29 - 51 
ninguna de ellas está claramente diferenciada respecto a la otra, por lo cual de manera gráfica es difícil asignar un peso a las remesas sobre la distribución del ingreso total.

Para determinar el efecto que tienen las remesas internacionales sobre la distribución del ingreso en la comunidad, se utiliza el método de descomposición del coeficiente de Gini. La tabla 4 consigna los resultados. Como ya había quedado explícito, las remesas internacionales representan un tercio del ingreso total en San Miguel ( $\mathrm{Sk}=0.32)$, y se distribuyen en mayor medida hacia hogares de mayor ingreso $(\mathrm{Gk}=0.80)$. Esta situación se verifica con la correlación que muestran las remesas al rango del ingreso total en los hogares $(\mathrm{Rk}=0.8186)$, que es muy alta. Los hogares de mayor ingreso capturan 80 centavos de cada peso de remesas que arriban a la comunidad. Cuando se conjuntan las tres medidas (SGR), se obtiene que las remesas contribuyen con el 55\% del valor que asume el coeficiente de Gini de la distribución del ingreso total comunal. Es decir, casi la mitad del valor del coeficiente de Gini del ingreso total se debe a las remesas. No obstante, una vez que al ingreso doméstico le sumamos el ingreso internacional, el coeficiente apenas empeora en $8 \%$ (pasa de 0.355 a 0.38 ; línea 4, columna 3 hasta línea 9 , columna 3). Esto sugiere que se da apenas un efecto visible negativo de las remesas internacionales, sobre la distribución del ingreso comunal, un efecto real pero insignificante en términos estadísticos. Este pequeño efecto puede verificarse en la gráfica 2. Ambas distribuciones del ingreso doméstico y del total se acompasan y sólo en la parte baja donde se ubican los hogares pobres, prácticamente son iguales. De la parte media hacia arriba, en los hogares de mayor ingreso las remesas empeoran la distribución. Parcialmente esto podría sugerir que con las remesas, los hogares de medio hacia arriba, se diferencian entre ellos, porque algunos reciben remesas y otros no, en cambio los hogares de menores ingresos se homogenizan en razón de que casi no reciben. De este modo, con las remesas se forman dos grandes grupos socioeconómicos, pero la des-

Tabla 3. Remesas y desigualdad según todas fuentes

\begin{tabular}{|c|c|c|c|c|c|}
\hline Fuente de ingreso & $\begin{array}{c}\text { \% en el in- } \\
\text { greso total }\end{array}$ & $\begin{array}{c}\text { C.Gini para } \\
\text { fuente de } \\
\text { ingreso } \\
\left(\mathrm{S}_{\mathrm{k}}\right)\end{array}$ & $\begin{array}{c}\text { Correlación Gini } \\
\text { de la fuente al } \\
\text { ingreso total } \\
\left(\mathrm{R}_{\mathrm{k}}\right)\end{array}$ & $\begin{array}{c}\text { Contribución al } \\
\text { C.Gini del in- } \\
\text { greso total } \\
\left(\mathrm{S}_{\mathrm{k}} \mathrm{G}_{\mathrm{k}} \mathrm{R}_{\mathrm{k}}\right)\end{array}$ & $\begin{array}{c}\text { \% proporcional } \\
\text { al Gini del in- } \\
\text { greso total }\end{array}$ \\
\hline Remesas & 0.3225 & 0.8049 & 0.8186 & 0.2125 & 0.55 \\
\hline Ingreso Doméstico & 0.6775 & 0.355 & 0.712387 & 0.171338 & 0.4463 \\
\hline Salarios & 0.4724 & 0.4353 & 0.5844 & 0.1202 & 0.31 \\
\hline Transferencias & 0.0360 & 0.6637 & 0.1095 & 0.0026 & 0.01 \\
\hline Negocios & 0.0607 & 0.8519 & 0.0958 & 0.0050 & 0.01 \\
\hline Acts_financieras & 0.0548 & 0.9349 & 0.6845 & 0.0351 & 0.09 \\
\hline Otro ingreso & 0.0536 & 0.6237 & 0.2539 & 0.0085 & 0.02 \\
\hline Ingreso total & $\mathbf{1}$ & $\mathbf{0 . 3 8 3 9}$ & $\mathbf{1}$ & $\mathbf{0 . 3 8 3 8}$ & $\mathbf{1}$ \\
\hline
\end{tabular}

Fuente: elaboración propia 
igualdad que se muestra ocurre entre los hogares ricos, aunque sus efectos se resienten también hacia los hogares pobres.

Estos resultados son semejantes con investigaciones que cuantifican valores similares para los mismos indicadores en otros lugares $(\mathrm{Sk}=0.16, \mathrm{Gk}=0.9, \mathrm{Rk}=0.86$ y SkGkRk=0.12), aunque en aquel, las remesas tienen una contribución de $30 \%$ al coeficiente de Gini, y un efecto más visible hacia el empeoramiento en la distribución del ingreso en la localidad de menor historia migratoria (Stark, Taylor and Yitzhaki, 1986). Nuestros resultados son compatibles también con otro trabajo que obtiene valores de ( $\mathrm{Gk}=0.41, \mathrm{Rk}=0.77)$, y una influencia de las remesas del 38\% en el valor del coeficiente de Gini total (Taylor and Wyatt, 1996). En relación con los valores que se presentan de manera agregada en similares indicadores $(\mathrm{Sk}=0.16, \mathrm{Gk}=0.87 \mathrm{y} \mathrm{Rk}=0.5)$, y una influencia neta de las remesas de $13 \%$ sobre el coeficiente de Gini total; la diferencia con nuestros resultados es marcada, en parte debida a que su muestra es de carácter nacional e incluye zonas de reciente y añeja tradición migratoria, lo cual lo hace incomparable (Taylor, Mora, Adams and López, 2005). No obstante, con la región Sur-Sureste, lugar donde se ubica San Miguel y que corresponde a una zona de reciente masificación migratoria, se presenta cierta similitud $(\mathrm{Sk}=0.14, \mathrm{Gk}=0.95$ y $\mathrm{Rk}=0.78)$ con una influencia de casi $17 \%$ en el coeficiente de Gini, y sobre todo un efecto negativo de las remesas mucho más contundente.

\section{La migración internacional, las remesas y las actividades agrícolas}

La forma específica y la intensidad con que la migración internacional y las remesas afectan las actividades agrícolas en San Miguel, se describe en la tabla 4. Los coeficientes que se exhiben son estadísticamente significativos. Es fácil apreciar que ni las remesas, ni la migración internacional ejercen ninguna influencia significativa sobre la contratación de peones en la comunidad. Ésta depende de factores endógenos. Las reservas del hogar y su composición interna, en cambio, ejercen mayor influencia. Por ejemplo, hogares con posesión de activos ganaderos e integrantes en situación de dependencia económica son quienes más demandan este trabajo en $\$ 0.03$ y $\$ 87$ pesos. En hogares con niños, se reduce la demanda de peones en casi $\$ 112$ pesos. Este resultado corrobora el reclamo de los estudios no económicos, en el sentido de que los infantes suplen a los migrantes, porque en estas comunidades no se promueve la demanda de trabajo agrícola. Igualmente, los hogares que reciben dólares destinados a financiar gastos de emigración, promueven la demanda de peones en $\$ 113$ pesos. Este resultado tiene sentido porque estos hogares tienen menores presiones económicas y la ubicación de recursos en la migración no reduce su capacidad productiva, pero también refleja el compromiso que los migrantes establecieron con sus hogares, al no desprotegerlo con la realización de este nuevo gasto. Con estas consideraciones, es previsible que los resultados encontrados en San Miguel sean diferentes a los efectos encontrados en estudios principalmente fuera de la visión económica de la migración, quienes trabajan en comunidades con fases diferentes o más avanzadas de migración y cuyos hallazgos su- 
Tabla 4. Resultados de la regresión

\begin{tabular}{|c|c|c|c|c|}
\hline \multicolumn{5}{|c|}{ Coeficientes significativos no estandarizados } \\
\hline & $\begin{array}{c}\text { Demanda de } \\
\text { Peones }\end{array}$ & $\begin{array}{l}\text { Volumen de } \\
\text { Producción }\end{array}$ & $\begin{array}{c}\text { Hectáreas en } \\
\text { Abandono }\end{array}$ & Ganadería \\
\hline Remesas que ingresan al hogar & $*$ & $*$ & $-0.0000052^{*}$ & $0.056^{* *}$ \\
\hline Ingresos domésticos del hogar & $*$ & $*$ & $-0.000058 * * * *$ & $*$ \\
\hline Valor del ganado & $0.03 * *$ & $-0.012 * *$ & & \\
\hline Compra de insumos & $-0.9467 * *$ & $0.4966^{* *}$ & & \\
\hline Renta de maquinaria & $-0.46 * *$ & $0.1987 * *$ & $0.0004^{*}$ & $-2.86^{*}$ \\
\hline Dólares gastados en vehículos & & $*$ & $-0.00018^{* *}$ & $*$ \\
\hline Riqueza en equipamiento del hogar & & $0.0044 * * * *$ & & \\
\hline Riqueza en vehículos & & $0.0013^{* *}$ & & \\
\hline Ingreso agrícola neto & $-0.689 * *$ & & & $5.7^{* *}$ \\
\hline Hectáreas propias del hogar & $*$ & $*$ & $0.217^{* *}$ & $*$ \\
\hline Hectáreas en abandono & $-32.93 *$ & $*$ & & \\
\hline Trabajo familiar & $*$ & $*$ & & $111.4^{*}$ \\
\hline Migración a USA por hogar & $*$ & $25.84^{* * * *}$ & $-0.585^{* *}$ & $*$ \\
\hline Educación de los migrantes & $*$ & $-9.65^{*}$ & & \\
\hline Niños & $-111.95^{* *}$ & $*$ & $*$ & \\
\hline Dependientes económicos & $87.27^{* *}$ & $*$ & & \\
\hline Contratación de peones & & $0.2782^{* *}$ & & \\
\hline Experiencia migratoria del hogar & & & $0.122^{* *}$ & $*$ \\
\hline Estado civil del migrante & & & $0.464^{* *}$ & \\
\hline Producción de maíz & $2.2955^{* *}$ & & & $-17.5^{* *}$ \\
\hline Producción de fríjol & $9.24 * *$ & & $0.005^{* * * *}$ & $-80.98 * *$ \\
\hline Consumo de fríjol & $-1.629 *$ & $0.5657^{*}$ & $-0.004 * * * *$ & \\
\hline Venta de terrenos y animales & $*$ & $*$ & $0.69 * * * *$ & $*$ \\
\hline Consumo de ahorros propios & $*$ & $*$ & $0.27^{*}$ & $*$ \\
\hline Recibo de dólares ex profeso & $138.14^{*}$ & $-81.27^{* *}$ & $-0.574 *$ & $1329.8^{* * * *}$ \\
\hline Constante & $16.399 * * *$ & $-40.69 * * * *$ & $0.071 * * *$ & $-210.2^{* * *}$ \\
\hline $\mathrm{R}_{\text {Ajustado }}^{2}$ & 0.78 & 0.907 & 0.684 & 0.6 \\
\hline $\mathrm{F}$ & 50.78 & 136 & 26.64 & 26.64 \\
\hline Probabilidad de ocurrencia * $p<0.05$ & $0<0.01 * * * * p<$ & & & \\
\hline
\end{tabular}

Fuente: elaboración propia.

brayan relaciones directas (Nava y Marrioni, 2003, p. 661), e inversas (Dinerman, 1982, p. 72; Fitting, 2004, p. 83; Wiest, 1983, p. 74) entre la migración internacional, las remesas y la demanda de trabajo en las comunidades. 
En relación con el volumen de producción, se puede resaltar que mientras éste es influido positivamente por la migración internacional que registran los hogares, no recibe ninguna influencia de las remesas. Aunque se trabajó con la misma variable de respuesta que usan los estudios económicos de la migración, ellos encuentran que la producción agrícola es afectada negativamente en un corto plazo por la salida de integrantes del hogar para labores de migración internacional y en mayor plazo se ve impulsada positivamente por las remesas generándose un incremento en el ingreso y el volumen de producción (Rozelle, Taylor and Brauw, 1999, p. 287). En San Miguel, es la migración lo que induce el efecto positivo en la producción, mientras que ésta permanece insensible a los efectos de las remesas internacionales. Aunque es previsible que tales efectos negativos de la migración y positivos de las remesas se presenten a futuro, conforme la comunidad acumule mayor experiencia migratoria y el rol que juega cada integrante del hogar en la actividad productiva, se defina con más claridad. Es decir, quien lleva a cabo la actividad agrícola realiza también otras. Por ejemplo, los coeficientes muestran que conforme una mayor cantidad de hogares financien la migración internacional de nuevos integrantes con remesas recibidas para tal propósito, y conforme los nuevos migrantes provengan del grupo de mayor educación, en esta comunidad la producción agrícola será negativa. A hora, la producción agrícola en San Miguel revela que los hogares con migrantes impulsan el incremento en la producción en casi $26 \mathrm{~kg}$, pero cuando su educación es superior al promedio, la producción se reduce en alrededor $10 \mathrm{~kg}$. Cuando el hogar financia la migración con dólares recibidos ex profeso, la producción se contrae hasta en $81 \mathrm{~kg}$. Este efecto negativo sugiere que la acumulación de migrantes en el hogar termina por minar sus actividades productivas agrícolas. Igualmente, se destaca que la compra de insumos, la renta de maquinaria y la riqueza del hogar fomentan la producción en pequeñas cantidades excepto por la ganadería -efecto negativo- que dada su naturaleza de traspatio, compite por los recursos en el hogar.

Por su parte, el abandono de tierras recibe un pequeño efecto negativo tanto de la migración internacional como de las remesas internacionales y de los ingresos domésticos del hogar. Como ya se observó, la teoría económica de la migración no tiene planteamiento al respecto, pero, al considerar las remesas como fuente de capital, se tenía la expectativa de que apoyaran el uso productivo de las tierras. Los coeficientes revelan que en esta comunidad, aunque en pequeñas cantidades, las tierras abandonadas se están reduciendo por el uso de las remesas internacionales, aunque igual efecto inducen otros ingresos del hogar. El coeficiente respectivo sugiere que la migración internacional beneficia en gran medida la labranza de las tierras en paro. Por cada migrante del hogar, se constata una reducción en las tierras en abandono en más de media hectárea. En cambio, la renta de maquinaria y la alta posesión de tierra fomentan el aumento de hectáreas cultivables en abandono. Con maquinaria en menor cantidad de tierra se pro46 ducen los alimentos. Igualmente, alta posesión de tierra induce a obviar la producción 
o a dejar en descanso alguna área cultivable. Los coeficientes también sugieren que, conforme los hogares acumulan experiencia migratoria y los nuevos migrantes son casados, el abandono de tierras crecerá en mayor proporción. Asimismo, la necesidad de alimentos en el hogar impulsa la recuperación de tierras aunque en menor proporción dada la disposición de éstos en las tiendas locales. En el caso de que el hogar venda algún activo -animal, terreno-, o consuma sus ahorros en financiar nuevas migraciones, las hectáreas en abandono tienden a aumentar entre 0.69 y $0.27 \mathrm{Ha}$; pero el efecto es revertido en mayor medida cuando tal gasto es asistido con dólares ex profeso.

En la Ganadería, las remesas internacionales inducen un efecto positivo, pero, en cambio, es insensible a la migración internacional. Al principio, la expectativa era que ésta fuera un medio de acumulación de remesas, similar a lo que se había detectado en otros lugares, incluso en perspectiva no económica (Mines, 1981, p. 60; Reichert, 1981, p. 63; Yunez, Taylor y Becerril, 2000; Lucas y Stark, 1985). Los resultados sugieren que en esta comunidad, por cada peso adicional de remesas que se recibe, el valor total de la ganadería aumenta en una proporción de 0.056 pesos, y que en aquellos hogares donde se financia la migración con remesas ex profeso, el valor de los activos de ganado aumenta más de 1.300 pesos. Esto recuerda que en San Miguel, como en otras regiones, los migrantes envían las remesas para ayudar a sus familias, pero también para atesorarlas en esta actividad. Igualmente, a medida que crece el volumen de producción el hogar tiene más posibilidades de alimentar su ganado -traspatio principalmente-, en consecuencia aumentan su posesión de animales. El trabajo familiar induce un efecto similar, mientras más días de trabajo dedican en sus actividades agrícolas, el atesoramiento en ganadería aumenta, en razón de la estrecha vinculación entre ganado de traspatio y producción agrícola. Cuantos más días de trabajo, se produce más alimento y se inducen mayores compras de animales. En contraste, la migración internacional, la edad de los migrantes y la experiencia migratoria de los hogares no inducen efectos sobre esta actividad. Igualmente, los gastos que realizan los hogares en reparación o construcción de la casa, así como los pagos que efectúan en maquinaria, empujan una menor inversión en compra de animales.

\section{Conclusiones}

La reciente historia migratoria de la comunidad y de acuerdo con el planteamiento de la Nueva Teoría Económica de la Migración, al inicio se conjeturó que las remesas internacionales afectarían negativamente la distribución del ingreso en San Miguel. En contraste, según los resultados obtenidos, no se encontró evidencia de que tal cosa estuviera ocurriendo. En el año de referencia -2004-, la comunidad resentía una distribución de ingresos un poco mejor que la que existía a nivel nacional de 0.46. La evidencia sugiere que la hipótesis de que los pueblos con historia migratoria reciente experimentan necesariamente marcada desigualdad en la distribución del ingreso ante 
el arribo de las remesas internacionales, debe ser discutida en función de la estructura y diversificación de ingresos que estructuralmente prevalece en la comunidad. La existencia de otras fuentes de ingresos en los hogares, pueden compensar y amortiguar en la comunidad los efectos de las remesas. Como se señala, en San Miguel la desigualdad que éstas registran proviene de la diferenciación económica que exhiben entre sí los hogares más ricos, aunque el efecto arrastra también los pobres.

En relación con las actividades agrícolas, está investigación revela que la salida de jóvenes y recursos económicos del hogar hacia la migración internacional, aunque sí reducen su capacidad productiva, no es ésta ni sus remesas los factores que determinan el desempeño de la actividad agrícola en ésta y, presumiblemente, en ninguna otra comunidad. Si bien, en los hogares se pueden apreciar relaciones positivas y negativas entre migración, remesas y demanda de trabajo agrícola (sobre todo aquellos con menos de tres migrantes que exhiben producción mayor), en las comunidades, al menos dos factores diluyen éstas: no hay un mercado laboral y los hogares cuentan con reserva de trabajadores. En este sentido, la demanda de trabajo agrícola depende más de variables endógenas (número de niños, dependientes económicos, posesiones de ganado, financiamiento de la migración), ni la migración internacional o las remesas pueden incidir sobre la estacionalidad y el patrón productivo de subsistencia de las actividades agrícolas, menos en la geografía de las tierras cultivables o en la irregularidad de las lluvias.

En relación con las tierras que se abandonan, este trabajo aporta evidencia de que la migración internacional y las remesas contribuyen a su recuperación, a diferencia del planteamiento que sostiene que éstas son variables que promueven su abandono. Aunque la propia geografía sinuosa de la comunidad podría alentar este efecto sobre todo en el largo plazo, conforme la experiencia migratoria del hogar se acumule. Un indicador relativo a los hogares es que, actualmente, sobre todo en aquellos con más de tres migrantes, las tierras son abandonadas. En la ganadería, se observa que con las remesas aumenta la acumulación de diversos ganados en los hogares, aunque la migración internacional por sí sola no altera esta variable. 


\section{Referencias}

Acevedo, G. (2003). La capacidad de los sectores económicos para la generación de empleos en la ciudad de Tlacolula de Matamoros. Tesis de maestría, ITO-Oaxaca.

Adams, R. Jr. (1989). "Worker Remittances and inequality in rural Egypt”, Economic Development and Cultural Change, 38(1).

Adelman, I., Taylor, E. y Stephen, V. (1988). "Life in a Mexican village: A SAM Perspective" En: The Journal of Development Studies, 25(1).

Ando, A. y Modigliani, F. (1963). “The Life cycle hypothesis of saving: aggregate implications and tests”. In: American Economic Review, 53(1). 55-84.

Cruz, C. (2002). La construcción de la Matriz de contabilidad social aplicada a Capulalpan de Méndez, Oaxaca y su utilización en el diseño de la política económica regional. Tesis doctoral. Universidad Autónoma de Chapingo.

Cohen, J. (2001). "Transnational migration in rural Oaxaca, Mexico: dependency, development and the household". In: American Anthropologist, 103(4).

Conapo (2002). Migración, remesas y desarrollo, Boletín sobre Migración, No. 19.

Cruz, Cuauhtémoc (2002). La construcción de la Matriz de contabilidad social aplicada a Capulalpan de Méndez, Oaxaca y su utilización en el diseño de la política económica regional. Tesis doctoral, Universidad Autónoma de Chapingo.

Díaz G. y Gretchen K. (2007). Mujeres Migrantes en Tránsito y Detenidas en México, Octubre: http://www.migrationinformation.org/Feature/display.cfm?id=648

De la Fuente, J. (1998). "La cultura zapoteca”, En Los zapotecos de la Sierra Norte de Oaxaca, en Ríos Manuel (comp.). Instituto Oaxaqueño de la cultura-CIESAS, Oaxaca.

Dinerman, I. (1982). Migrants and stay-at-homes: a comparative study of rural migration from Michoacán México. Monograph series 5, Center for U.S.-Mexican Studies, University of California, San Diego.

Dinerman, I. (1983). “El impacto agrario de la migración en Huecorio”. En: Relaciones, 4(15). Zamora Michoacán, Colmich.

Djajic, S. (1986). "Internacional migration, remitances and welfare in a dependent economy". In: Journal of Development Economics, 21.

Fitting, L. (2004). "No hay dinero en la milpa": el maíz y el hogar trasnacional del sur del Valle de Tehuacan”. En: Binford Leigh (ed). La economía política de la migración internacional en Puebla y Veracruz: siete estudios de caso. Puebla: BUAP.

Hernández, J. (2001). Artesanías y artesanos en Oaxaca. Innovaciones de la tradición. México: CONACULTA-Fonca.

INEGI, Encuesta nacional de ingreso gasto (ENIGH). Varios años.

Lerman, R. y Yitzhaki, S. (1985). "Income inequality effects by income source: a new approach and applications to the united states". In: Review of Economics and Statistics, (67)1.

Lerman, R. y Yitzhaki, S. (1995). "Changing ranks and the inequality impacts of taxes and transfers”. In: National Tax Journal, (48)1.

Lipton, M. (1980). "Migration from rural areas of poor countries. The impact on rural productivity and income distribution". In: World Development, 1(8). 
López, G. (1988). “La migración a Estados Unidos en Gomes Farías Michoacán”, en López Gustavo y Pardo Galván Sergio (Edits.). Migración en el Occidente de México, Zamora Michoacán, México, Colmich.

López, F. y Runsten, D. (2004). "El trabajo de los mixtecos y los zapotecos en California: experiencia rural y urbana”, en Fox J. y Rivera G. (coords.). Indígenas mexicanos migrantes en los Estados Unidos, México: Cámara de Diputados, LIX legislatura-The University of California-Universidad Autónoma de Zacatecas-Miguel Ángel Porrua.

Lucas, R. (1987). "Emigration South Africa's mines". In: The American Economic Rewiev, $3(77)$.

Lucas, R. and Stark O. (1985). "Motivations to remit: evidence from Botswana". In: Journal of Political Economy, 93.

Mines, R. (1981). Developing a community tradition of migration: a field study in rural Zacatecas, Mexico and California settlement areas. In: Monographs in U.S.-Mexican Studies, No. 3, Program in United States-Mexican Studies. University of California San Diego, La Jolla California

Nava, T. y Marrioni (2003). “El impacto de la migración en la actividad agropecuaria en Petlalcingo Puebla, Agrociencia, (37)6.

Quiroz Zúñiga, Pérez Soto, Barrios Puente (2000). "Migración a Estados Unidos y desarrollo en Loma Tendida municipio de Valle de Santiago Guanajuato". En: Lenin Navarro y Vargas Uribe (coords.). El impacto económico de la migración en el desarrollo regional de México. Estudios de caso de los estados de Guanajuato, Michoacán y Zacatecas. Morelia Michoacán: Escuela de Economía, Universidad Michoacana de San Nicolás de Hidalgo,

Pérez, L. y David C. (2009). "Migrantes, viaje al infierno del secuestro”. En: Revista Contralinea, México, 30 agosto.

Ramírez E. (2009). "México: 10 mil migrantes secuestrados en 6 meses”. En: Revista Contralinea. 30 agosto. http://www.contralinea.info/archivo-revista/index.php/2009/08/30/ mexico-10-mil-migrantes-secuestrados-en-6-meses/

Rionda, L. (1992). Y jalaron pa'l norte. INI-Colmich.

Reichert, J. (1981). "The migrant syndrome: seasonal U.S wage labour and rural development in central Mexico". In: Human Organization, 40(1).

Reyes et al., (2001). Impacto de las remesas internacionales en el desarrollo de las localidades expulsoras de población en Oaxaca, México. Reporte presentado a UC Mexus - CONACyT. Enero.

Rozelle, S., Taylor, E. y Alan de B. (1999). "Migration, remittances, and agricultural productivity in China". In: American Economic Review, 89(2).

Stark, O. (1982). "Research on rural to urban migration in LDCs: the confusion frontier and why we should pause to rethink afresh". In: World development, 10(1). Gran Bretaña.

Stark, O. y David, B. (1985). “The New Economics of Labor Migration”. In: American Economic Review, 75, 173-78.

Stark, O., Taylor, E. y Shlomo, Y. (1986). "Remittances and inequality”. In: The Economic Journal, (96). 
Taylor, E. y Wyatt (1996). "The shadow value of migrant remittances, income and inequality in a household farm economy". In: The Journal of Development Studies, (32)6.

Taylor, E. (1995). Micro economic-wide models for migration and policy analysis: an application to rural Mexico. Paris: OECD.

Taylor, E., Mora, A. y Lopez, (2005). Remittances, inequality and poverty: evidence from rural Mexico. American Agricultural Economics Association Annual Meeting. Providence: Rhode Island, July 24-27.

Wiest, R. (1973). "Wage-labor migration and the household in a Mexican town". In: Journal of Anthropological Research, Vol. 29.

Wiest, R. (1984). "La dependencia externa y la perpetuación de la migración temporal a Estados Unidos. En: Relaciones, 4,(15). Zamora Michoacán, Colmich.

Yunez, A., Taylor, E. y Becerril, J. (2000). "Los pequeños productores rurales en México: características y análisis de impactos”. En: Yunez Naude (comp.), Los pequeños productores rurales en México: las reformas y las opciones. México: Colmex. 
